\title{
An Improved On-the-Fly Tableau Construction for a Real-Time Temporal Logic
}

\author{
Marc Geilen \\ Dept. of Elec. Eng., Eindhoven University of Technology, The Netherlands \\ m.c.w.geilen@tue.nl
}

\begin{abstract}
Temporal logic is popular for specifying correctness properties of reactive systems. Real-time temporal logics add the ability to express quantitative timing aspects. Tableau constructions are algorithms that translate a temporal logic formula into a finite-state automaton that accepts precisely all the models of the formula. On-the-fly versions of tableau-constructions enable their practical application for modelchecking. In a previous paper we presented an on-the-fly tableaux construction for a fragment of Metric Interval Temporal Logic in dense time. The interpretation of the logic was constrained to a special kind of timed state sequences, with intervals that are always left-closed and right-open. In this paper we present a non-trivial extension to this tableau construction for the same logic fragment, that lifts this constraint.
\end{abstract}

\section{Introduction}

Temporal logic 13 is a popular formalism for expressing properties of reactive systems. Finite state models of such programs can be analysed automatically with model checking procedures. Temporal logics exist in different flavours. In particular, real-time temporal logics have been developed for expressing timing aspects of systems. A tableau construction is an algorithm that translates a temporal logic formula into a finite-state automaton that accepts precisely all the models of the formula. The automata-theoretic approach to model checking [12 14] employs tableau algorithms to turn a temporal formula into an observer of a system's behaviours. For practical applications, tableau constructions are being improved and optimised (e.g., [569]). An important improvement has been the development of on-the-fly versions of tableau constructions. Such onthe-fly tableau constructions are based on a normal form for temporal formulas in which the constraints on the current state are separated from the constraints on the future states. In the real-time domain, tableau constructions have been developed for various logics and their complexities have been studied [210]. For dense time linear temporal logic the algorithms of 24 and 8 are (to the best of the author's knowledge) the only existing tableau constructions to date. [24] being aimed at establishing a theoretical connection between temporal logic and timed automata and [8] a first attempt at a practical algorithm suitable for model checking. [1] describes the construction of so-called testing automata from 
formulas in a safety modal logic for the UPPAAL tool. The logic is a restricted variant of a real-time extension of Hennessy-Milner logic.

Towards the creation of an efficient tableau construction for dense real-time, we presented in [8] a first version of an on-the-fly tableau construction for a linear temporal logic with dense time. A drawback of this work is that formulas are interpreted over a restricted kind of state sequences. The logic considered both in that paper and this paper, is based on a fragment of Metric Interval Temporal Logic (MitL, see 44) that is decidable in PSPACE. In this paper we remove the restriction of 8 and show that, at the expense of some extra bookkeeping and hence some more locations in the tableaux, we can apply the similar ideas to obtain an on-the-fly tableau construction for a dense-time linear temporal logic in the traditional interpretation, enabling the connection to common theory and tools. We also demonstrate a small flaw in the classical result of [2,4, a tableau construction for MiTL, that can be solved with techniques introduced in this paper. The new tableau algorithm is also based on a normal form procedure for temporal logic formulas, that separates constraints on the current interval from constraints on the remainder of the state sequence. In order to deal with arbitrary interval types, two normal forms are considered related to two kinds if time intervals, singular and open intervals. In order to define these normal forms, the logic is extended with timers that can be explicitly set and tested, and with a Next operator referring to the beginning of the next time interval.

Section 2 introduces the logic and timed automata that we use. Section 3 discusses the main ideas behind the tableau construction. Section 4 presents the normal form for formulas on which the tableau algorithm of Section 5 is based. Section 6 concludes.

\section{Preliminaries}

An $(\omega$ - $)$ word $\bar{w}=\sigma_{0} \sigma_{1} \sigma_{2} \ldots$ over an alphabet $\Sigma$ is an infinite sequence of symbols from $\Sigma ; \bar{w}(k)$ denotes $\sigma_{k}$ and $\bar{w}^{k}$ refers to the tail $\sigma_{k} \sigma_{k+1} \sigma_{k+2} \ldots$ We use the latter notations for other kinds of sequences as well. An interval $I$ is a convex subset of $\mathbb{R}^{\geq 0}$; it has one of four shapes: $[a, b],[a, b),(a, b]$ or $(a, b) . l(I)(r(I))$ denotes the lower (upper) bound $a(b)$ and $|I|$ the length of $I$. We use $I-t$ to denote the interval $\left\{t^{\prime}-t \in \mathbb{R}^{\geq 0} \mid t^{\prime} \in I\right\}$. An interval sequence $\bar{I}=I_{0} I_{1} I_{2} \ldots$ is an infinite sequence of intervals that are adjacent, meaning $r\left(I_{i}\right)=l\left(I_{i+1}\right)$ and that are of matching type (right-closed and left-open or right-open and left-closed) for every $i$, for which $l\left(I_{0}\right)=0$, and which is diverging, i.e. any $t \in \mathbb{R}^{\geq 0}$ belongs to precisely one interval $I_{i}$. A timed word $\bar{u}$ over $\Sigma$ is a pair $(\bar{w}, \bar{I})$ consisting of an $\omega$-word $\bar{w}$ over $\Sigma$ and an interval sequence $\bar{I}$. For $t \in \mathbb{R}^{\geq 0}, \bar{u}(t)$ denotes the symbol present at time $t$, this is $\bar{w}(k)$ if $t \in \bar{I}(k)$. For such a $t$ and $k, \bar{u}^{t}$ is the tail of the timed word, consisting of the word $\bar{w}^{k}$ and of the interval sequence $\bar{I}(k)-t, \bar{I}(k+1)-t, \ldots$. The timed word determines a mapping from $\mathbb{R}^{\geq 0}$ to $\Sigma$. Timed words $\bar{u}_{1}$ and $\bar{u}_{2}$ are equivalent (denoted $\bar{u}_{1} \equiv \bar{u}_{2}$ ) if they define the same mapping, i.e. for all $t \geq 0, \bar{u}_{1}(t)=\bar{u}_{2}(t)$. A timed state sequence (TSS) over a set Prop of propositions is a timed word over the alphabet $2^{\text {Prop }}$. 


\subsection{Real-Time Temporal Logic}

We consider a restricted version of the real-time temporal logic Mith of [4, $\mathrm{MITL}_{\leq}$, with formulas of the following form $(d \in \mathbb{N})$.

$$
\varphi::=\text { true }|p| \neg \varphi\left|\varphi_{1} \vee \varphi_{2}\right| \varphi_{1} \mathrm{U}_{\leq d} \varphi_{2}
$$

Formulas of this form are called basic, in order to distinguish them from formulas using an extended syntax that is to be defined in Section 4.1. A formula is interpreted over a timed state sequence $\bar{\rho}$ as follows.

$$
\begin{array}{ll}
\bar{\rho} \models \text { true } & \text { for every timed state sequence } \bar{\rho} ; \\
\bar{\rho} \models p & \text { iff } p \in \bar{\rho}(0) ; \\
\bar{\rho} \models \neg \varphi & \text { iff not } \bar{\rho} \models \varphi ; \\
\bar{\rho} \models \varphi_{1} \vee \varphi_{2} & \text { iff } \bar{\rho} \models \varphi_{1} \text { or } \bar{\rho}=\varphi_{2} ; \\
\bar{\rho} \models \varphi_{1} \mathrm{U}_{\leq d} \varphi_{2} & \text { iff there is some } 0 \leq t \leq d, \text { such that } \bar{\rho}^{t} \models \varphi_{2} \text { and } \\
& \text { for all } 0 \leq t^{\prime}<t, \bar{\rho}^{\prime} \models \varphi_{1} .
\end{array}
$$

Note that no basic $\mathrm{MITL}_{\leq}$formula can distinguish equivalent timed state sequences. We also use the duals: $\varphi_{1} \wedge \varphi_{2}=\neg\left(\neg \varphi_{1} \vee \neg \varphi_{2}\right)$, and Release $\varphi_{1} \vee_{\leq d} \varphi_{2}=$ $\neg\left(\neg \varphi_{1} \mathrm{U}_{\leq d} \neg \varphi_{2}\right)$. The restriction of the bound $d$ to finite naturals simplifies the presentation as it yields a logic in which only safety properties can be expressed, thus avoiding the need to handle acceptance conditions. It is straightforward to extend our results using standard techniques (see e.g., 9] ) to a logic which also includes an unbounded Until operator. Also other types of bounds $(<d, \geq d$, $>d$ ) should be easy to add.

$\boldsymbol{\varphi}$-Fine Interval Sequences In the on-the-fly tableau constructions of [5] for untimed logics, a formula is rewritten so that the constraints on the current state are separated from those on the remainder of the state sequence. This is possible because of the discrete nature of the sequence. In the dense time case, there is no such thing as a next state. The discretisation suggested by the interval sequence $\bar{I}$ of a timed state sequence $\bar{\rho}$ is, in general, not fine enough for our purposes: when interpreted over tails of $\bar{\rho}$, the truth value of a formula may vary along a single interval of $\bar{I}$.

Definition 1. ([28]) Let $\varphi \in \mathrm{MITL}_{\leq}$. An interval sequence $\bar{I}$ is called $\varphi$-fine for timed state sequence $\bar{\rho}$ if for every syntactic subformula $\psi$ of $\varphi$, every $k \geq 0$, and every $t_{1}, t_{2} \in \bar{I}(k)$, we have $\bar{\rho}^{t_{1}} \models \psi$ iff $\bar{\rho}^{t_{2}} \models \psi$. In case that $\bar{I}$ is $\varphi$-fine for a timed state sequence $(\bar{\sigma}, \bar{I})$, also $(\bar{\sigma}, \bar{I})$ will be called $\varphi$-fine.

In [2], Lemma 4.11 it is shown that the intervals of any timed state sequence can always be refined to be fine for any Mitu formula. Being a subset of MiTL, the same holds for MiTL $_{\leq}$. Since every refinement (by splitting intervals) of a $\varphi$-fine interval sequence is again a $\varphi$-fine interval sequence, it follows that there must also exist a $\varphi$-fine interval sequence consisting of only singular and open intervals [2]. In the remainder of the paper we will assume (without loss of generality) that every interval sequence $\bar{I}$ consists of alternating singular and open intervals, i.e. $\bar{I}(0)$ is singular, $\bar{I}(1)$ is open, $\bar{I}(2)$ is singular, and so on. 


\subsection{Timed Automata}

The target of our tableau construction are timed automata in the style of Alur and Dill [3. We use a variation, adapted to our needs, that can easily be translated to Alur-Dill automata. We would like to control the type of interval transitions (open to closed or closed to open). Therefore, we use two kinds of locations, locations $\left(L_{s}\right)$ in which the automaton may only reside for a single instant and locations $\left(L_{o}\right)$ in which the automaton may only reside for intervals that are both left-open and right-open. The automaton must necessarily alternate both kinds of locations. This behaviour can be easily enforced in a standard timed automaton (see e.g., 2]). A location can only be visited in singular intervals if a timer is set to 0 upon entering the location and is required to be equal to 0 while staying in that location. Locations visited in between singular intervals can then only correspond to open intervals. Moreover, the automata use timers that decrease as time advances, possibly taking negative values in $\mathbb{R}$ (this is to facilitate the correspondence with temporal logic formulas). They may be set to nonnegative integer values and may be compared to zero only. Given a set $T$ of timers, a timer valuation $\nu \in T \operatorname{Val}(T)$ is a mapping $T \rightarrow \mathbb{R}$. For $t \in \mathbb{R}^{\geq 0}, \nu-t$ denotes the timer valuation that assigns $\nu(x)-t$ to any timer $x$ in the domain of $\nu$. A timer setting $T S \in T S e t(T)$ is a partial mapping $T \rightarrow \mathbb{N}$. We use $T S[x:=d]$ (where $x \in T$ and $d \in \mathbb{N}$ ) to denote the timer setting that maps $x$ to $d$ and other timers to the same value as $T S .[x:=d]$ is short for $\varnothing[x:=d]$. For a timer valuation $\nu$ and a timer setting $T S, T S(\nu)$ is the timer valuation that maps any timer $x$ in the domain of $\nu$ to $T S(x)$ if defined, and to $\nu(x)$ otherwise. The set TCond $(T)$ of timer conditions over $T$ is $\{x>0, x \geq 0, x<0, x \leq 0 \mid x \in T\}$.

Definition 2. Let $\Sigma$ be an alphabet. $A$ timed automaton $A=\left\langle L_{s}, L_{o}, T, L_{0}, Q\right.$, $T C, E\rangle$ over $\Sigma$ consists of

- a finite set $L=L_{s} \cup L_{o}$ of locations $\ell$ that are either singular $\left(\ell \in L_{s}\right.$ ) or open $\left(\ell \in L_{o}\right)$;

- a set $T$ of timers;

- a finite set $L_{0}$ of initial extended locations $\left(\ell_{0}, \nu_{0}\right) \in L_{s} \times T \operatorname{Val}(T)$, where $\nu_{0}$ assigns integer values to the timers;

- a mapping $Q: L \rightarrow 2^{\Sigma}$ labelling every location with a set of symbols from the alphabet;

- a mapping TC $: L \rightarrow 2^{\text {TCond }(T)}$ labelling every location with a set of timer conditions over $T$;

- a set $E \subseteq L \times T S e t(T) \times L$ of edges labelled by timer settings, such that for every edge $\left\langle\ell, T S, \ell^{\prime}\right\rangle \in E$, if $\ell \in L_{s}$ then $\ell^{\prime} \in L_{o}$ and if $\ell \in L_{o}$ then $\ell^{\prime} \in L_{s}$.

An extended location $\lambda$ is a pair $(\ell, \nu)$ consisting of a location $\ell$ and a timer valuation $\nu$. For an automaton with the set $T$ of timers, we use $\overline{0}$ to denote the timer valuation that maps every timer in $T$ to 0 . A timed run describes the path taken by the automaton when accepting a timed word. It gives the location of the automaton and the values of its timers at any moment, by recording the sequence of locations, the intervals during which the automaton resides in those locations, and the timer values at the time of transition to the interval. 
Definition 3. A timed run $\bar{r}$ of an automaton $\left\langle L_{s}, L_{o}, T, L_{0}, Q, T C, E\right\rangle$ is a triple $(\bar{\ell}, \bar{I}, \bar{\nu})$ consisting of a sequence of locations, an interval sequence, alternating singular and open intervals, and a sequence of timer valuations, such that: - [Consecution] for all $k \geq 0$, there is an edge $\left(\bar{\ell}(k), T S_{k}, \bar{\ell}(k+1)\right) \in E$ such that $\bar{\nu}(k+1)=T S_{k}(\bar{\nu}(k)-|\bar{I}(k)|)$;

- [Timing] for all $k \geq 0$ and $t \in \bar{I}(k)$, the timer valuation at time $t, \bar{\nu}(k)-$ $(t-l(\bar{I}(k)))$, satisfies all timer conditions in $T C(\bar{\ell}(k))$.

We write $\bar{r}(t)$ to denote the location of $\bar{r}$ at time $t$, i.e. $\bar{\ell}(k)$ if $t \in \bar{I}(k)$. Given a timed word $\bar{u}, \bar{r}$ is a run for $\bar{u}$ if

- [Symbol match] for all $t \geq 0, \bar{u}(t) \in Q(\bar{r}(t))$.

$A$ accepts $\bar{u}$ if it has a run for $\bar{u}$ from an initial extended location. The timed language $\mathcal{L}(A)$ of $A$ is the set of all timed words that it accepts.

It follows from Def. 3 that the language of a timed automaton is closed under equivalence. This will allow us to restrict our attention to $\varphi$-fine sequences, alternating singular and open intervals, when arguing the completeness of the tableau construction for a basic $\mathrm{MITL}_{\leq}$formula $\varphi$.

\section{Towards a Tableau Construction}

In the tableau construction, we are going to use timers to remember when certain relevant transitions have been made and to enforce time constraints between such transitions. Unfortunately, recording the time of transition alone is not enough, it is also necessary to remember in what fashion the transition was performed, from a right-open to a left-closed interval or from a right-closed to a left-open one. This can be demonstrated using Fig. [ 1 we see the beginning of a timed state sequence with interval sequence $[0,2](2,4](4, \ldots$ and state sequence $\varnothing \varnothing\{p\} \ldots$ and the formula $\nabla_{\leq 2} p$ (short for trueU $U_{\leq 2} p$ ). It is easy to check that the first intervals of the timed state sequence are $\nabla_{<2} p$-fine and that $\nabla_{<2} p$ does not hold in the first interval and does hold in the second and third. If we imagine the tableau automaton trying to accept this TSS and checking that $\nabla_{\leq 2} p$ holds in the second interval (which is left-open), it would need to set a timer, say $x$, at time 2, the time of the transition. Now it must check that a state is reached where $p$ holds within 2 units of time. To be precise, as it is the case in Fig. 1 $p$ must hold at the latest in some left-open interval starting at time instant 4 . This would have been different, however, if the transition at time 2 were from a right-open to a left-closed interval, in that case, $\mathrm{p}$ must hold at the latest at time instant 4 itself. This example shows that one must record not only the time of the transition, but also its type. To deal with this issue, 224] distinguishes singular and open intervals. However, we believe that the tableau construction of 24] does not deal with the former situation correctly, in this case it requires only that $p$ holds at the latest at the instant 4 itself and does not allow for $p$ to become true immediately after that 1 ; this can be resolved using the technique outlined in this paper.

\footnotetext{
${ }^{1}$ Assume (using notation of [2,4]) we have the MITL formula $\psi=\diamond_{(0,1]} p$. Consider an incoming edge to an open-interval location containing $\psi$ from a location not
} 


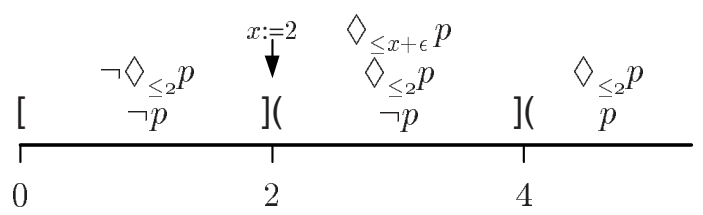

Fig. 1. Checking the formula $\diamond_{\leq 2} p$

Fig. 1 also shows how a tableau automaton can deal with checking the formula $\nabla_{\leq 2} p$. At the beginning of the interval, a timer $x$ is set to 2 . The type of transition is recorded in the form of the constraint that is remembered during the subsequent interval. This constraint, $\diamond_{\leq x+\epsilon} p$, represents the fact that during the interval (while $x$ decreases), for any $\epsilon>0$, there must be some point in time, at most $x+\epsilon$ units of time away, where $p$ holds. We introduce a number of constructs that we will later learn how to interpret as formulas themselves and are needed to define the tableau automata.

Definition 4. With an Until formula $\psi=\varphi_{1} \mathrm{U}_{\leq d} \varphi_{2}$ we associate the set:

$$
\Xi^{\psi}=\left\{\varphi_{1} \mathrm{U}_{\leq x_{\psi}} \varphi_{2}, \varphi_{1} \mathrm{U}_{\leq x_{\psi}+\epsilon} \varphi_{2}, x_{\psi}>0, x_{\psi} \geq 0\right\}
$$

and with a Release formula $\psi=\varphi_{1} \mathrm{~V}_{\leq d} \varphi_{2}$ we associate the set:

$$
\Xi^{\psi}=\left\{\varphi_{1} \mathrm{~V}_{<d} \varphi_{2}, \varphi_{1} \mathrm{~V}_{<x_{\psi}} \varphi_{2}, \varphi_{1} \mathrm{~V}_{\leq x_{\psi}} \varphi_{2}, x_{\psi}<0, x_{\psi} \leq 0\right\}
$$

The closure $\operatorname{cl}(\varphi)$ of an $\mathrm{MITL}_{\leq}$formula $\varphi$ in positive form is the smallest set $\Phi$ such that

- every syntactic subformula of $\varphi$, including $\varphi$ itself, is in $\operatorname{cl}(\varphi)$;

- for every Until or Release formula $\psi \in \operatorname{cl}(\varphi), \Xi^{\psi} \subseteq \operatorname{cl}(\varphi)$.

In untimed tableau constructions, the constraints on the current state are separated from the constraints on the remainder of the state sequence. In the tableau for real-time temporal logic with only one kind of intervals of [8], we mimicked this by separating constraints on the first interval from constraints on the timed state sequence starting from the second interval. In this case, we try the same. However, the constraints that need to be imposed on the next interval now depend on the type of transition as well. Fig. 2 shows another example. If we have to check that $\varphi_{1} \mathrm{~V}_{\leq d} \varphi_{2}$ holds during some initial interval of length 2 , and we know that $\varphi_{2}$ holds during this interval but we do not know that $\varphi_{1}$ holds, then at the end of the interval we need to set a timer to $d$ and check that $\varphi_{2}$ remains

containing $x_{\psi}$. Then the timer is allowed to be reset upon entering the location. From that point onwards, subsequent locations contain $x_{\psi}$ and the constraint $x_{\psi} \in(0,1]$ until a location is reached containing $p$ and $x_{\psi} \in(0,1]$ and the timer $x_{\psi}$ is not reset in the mean time. Thus by the time when $x_{\psi}=1$, a location containing $p$ must have been reached, otherwise the run is stuck. But the formula holds in the initial open interval if $p$ becomes true immediately after this moment. 

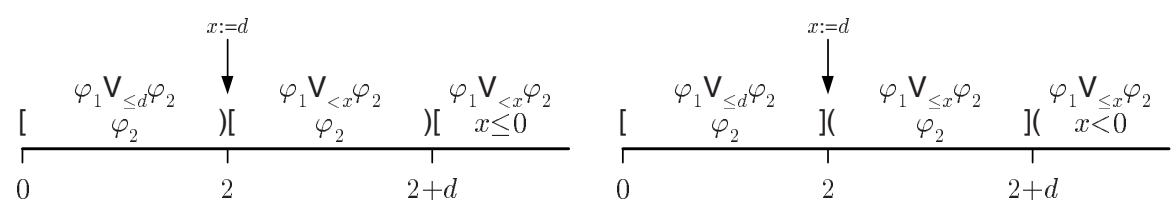

Fig. 2. Separating constraints for the next interval depending on transition type

to hold until either the timer expires or $\varphi_{1}$ holds. How long exactly, $\varphi_{2}$ needs to hold depends again on the type of transition. If it is from a right open to a left closed interval (Fig. 22, left), then the formula $\varphi_{1} \mathrm{~V}_{\leq d} \varphi_{2}$ need not hold at the time of the transition itself and therefore, $\varphi_{2}$ is only required to hold up to but not including the point where the timer expires, hence the constraint $\varphi_{1} \mathrm{~V}_{<x} \varphi_{2}$. If the transition is from a right-closed to a left open interval (see Fig. 2, right), then the formula $\varphi_{1} \mathrm{~V}_{\leq d} \varphi_{2}$ must also hold at the time of the transition and $\varphi_{2}$ must still hold at the point where the timer expires, hence the constraint $\varphi_{1} \mathrm{~V}_{\leq x} \varphi_{2}$.

\section{A Disjunctive Temporal Normal Form}

On-the-fly tableau constructions are generally based on rewriting formulas in some disjunctive temporal normal form that separates constraints on the current moment from constraints on the future and that orders disjunction and conjunction so that disjunctions can be mapped to non-deterministic choice of the automaton. In [8], we used intervals for the discretisation of the time-domain that allows to effectively separate 'now' from 'the future'. For this we introduced a $\bigcirc$ operator that supported this for the type of intervals that we used. Now we want to repeat this approach for the interval sequences of alternatingly singular and open intervals. We essentially present two normal form rewrite procedures, one for each interval type, that preserve equivalence of the formula throughout that interval. To this end, we start with two satisfaction relations. The first, denoted $\stackrel{s}{=}$, for singular intervals, is in fact the ordinary satisfaction relation $\models$, but is furthermore defined for the extended formulas introduced in the next section. The second, $\stackrel{\varrho}{=}$, asserts that a formula holds during the first open interval.

\subsection{Extending the Logic}

In order to define the normal form towards the construction of timed automata, the logic is extended with timers that can be bound by timer setting operators or which can be free. A formula is interpreted in the context of a timer environment which provides a value for every free timer. These timers will be related to timers of the timed tableau automaton. Besides that, we introduce a Next operator $(\bigcirc)$, that will form the basis for deriving its transitions. Moreover we assume that formulas are in positive normal form (negations only occur in front of propositions) using the dual operators (false, $\wedge, \mathrm{V}$ ). The timer set operator 
can set timers to integer values, and timers can be compared to 0 only by checking the conditions $x>0, x \geq 0, x<0$ or $x \leq 0$. The arguments of an Until or Release operator must be basic $\mathrm{MITL}_{\leq}$formulas.

$\mathrm{MITL}_{\leq}$is redefined. Besides the basic formulas defined in section 2 we add formulas of the following form, where $\varphi, \varphi_{1}$, and $\varphi_{2}$ are basic formulas, $d \in \mathbb{N}$, $T S$ is a timer setting, $x$ is a timer and $\sim \in\{<, \leq,>, \geq\}$.

$$
\begin{gathered}
\psi::=\varphi\left|\psi_{1} \vee \psi_{2}\right| \psi_{1} \wedge \psi_{2}|T S . \psi| x \sim 0\left|\varphi_{1} \mathrm{~V}_{<d} \varphi_{2}\right| \varphi_{1} \mathrm{U}_{\leq x} \varphi_{2} \mid \varphi_{1} \mathrm{U}_{\leq x+\epsilon} \varphi_{2} \\
\left|\varphi_{1} \mathrm{~V}_{<x} \varphi_{2}\right| \varphi_{1} \mathrm{~V}_{\leq x} \varphi_{2} \mid \bigcirc \psi
\end{gathered}
$$

The semantics is extended as follows. We write $\bar{\rho} \mid \stackrel{s}{=}{ }_{\nu} \psi$ to denote that the timed state sequence $\bar{\rho}$ satisfies $\psi$ in the context of the timer valuation $\nu$.

$$
\begin{aligned}
& \bar{\rho} \mid \stackrel{s}{=}_{\nu} \varphi \quad \text { iff } \quad \bar{\rho}=\varphi \text {; } \\
& \bar{\rho} \stackrel{s}{=}{ }_{\nu} \psi_{1} \vee \psi_{2} \quad \text { iff } \quad \bar{\rho} \stackrel{s}{=}{ }_{\nu} \psi_{1} \text { or } \bar{\rho} \stackrel{s}{=}{ }_{\nu} \psi_{2} \text {; } \\
& \bar{\rho} \stackrel{s}{=}{ }_{\nu} \psi_{1} \wedge \psi_{2} \quad \text { iff } \quad \bar{\rho} \longmapsto \stackrel{s}{=}_{\nu} \psi_{1} \text { and } \bar{\rho} \longmapsto{ }^{=}{ }_{\nu} \psi_{2} \text {; } \\
& \bar{\rho} \stackrel{s}{=}{ }_{\nu} \varphi_{1} \vee_{<d} \varphi_{2} \quad \text { iff } \quad \text { for all } 0 \leq t<d, \bar{\rho}^{t} \stackrel{s}{=}_{\nu-t} \varphi_{2} \text { or there is some } 0 \leq \\
& t^{\prime}<t \text {, such that } \bar{\rho}^{t^{\prime}} \stackrel{s}{=}{ }_{\nu-t^{\prime}} \varphi_{1} \text {; } \\
& \bar{\rho} \stackrel{s}{=}{ }_{\nu} T S . \psi \quad \text { iff } \quad \bar{\rho} \stackrel{s}{=}_{T S(\nu)} \psi \text {; } \\
& \bar{\rho} \stackrel{s}{=}{ }_{\nu} \varphi_{1} \mathrm{U}_{\leq x} \varphi_{2} \quad \text { iff } \quad \bar{\rho} \stackrel{s}{=}{ }_{\nu} \varphi_{2} \text { or there is some } 0 \leq t \leq \nu(x) \text {, such that } \\
& \bar{\rho}^{t} \stackrel{s}{=}_{\nu-t} \varphi_{2} \text { and for all } 0 \leq t^{\prime}<t, \bar{\rho}^{t^{\prime}} \stackrel{s}{=}_{\nu-t^{\prime}} \varphi_{1} \text {; } \\
& \bar{\rho} \stackrel{s}{=}{ }_{\nu} \varphi_{1} \mathrm{U}_{\leq x+\epsilon} \varphi_{2} \text { iff } \bar{\rho} \stackrel{s}{=}{ }_{\nu} \quad \varphi_{2} \text { or for every } \epsilon>0 \text {, there is some } \\
& 0 \leq t \leq \nu(x)+\epsilon \text {, such that } \bar{\rho}^{t} \stackrel{s}{=}_{\nu-t} \varphi_{2} \text { and for } \\
& \text { all } 0 \leq t^{\prime}<t,\left.\bar{\rho}^{t^{\prime}}\right|^{=}{ }_{\nu-t^{\prime}} \varphi_{1} \text {; } \\
& \bar{\rho} \stackrel{s}{=}{ }_{\nu} \varphi_{1} \mathrm{~V}_{<x} \varphi_{2} \quad \text { iff } \quad \text { for all } 0 \leq t<\nu(x),\left.\bar{\rho}^{t}\right|^{\underline{s}}{ }_{\nu-t} \varphi_{2} \text { or there is some } \\
& 0 \leq t^{\prime}<t \text {, such that } \bar{\rho}^{t^{\prime}} \stackrel{s}{=}{ }_{\nu-t^{\prime}} \varphi_{1} \text {; } \\
& \bar{\rho} \stackrel{s}{=}{ }_{\nu} \varphi_{1} \mathrm{~V}_{\leq x} \varphi_{2} \quad \text { iff } \quad \text { for all } 0 \leq t \leq \nu(x),\left.\bar{\rho}^{t}\right|_{=}{ }_{\nu-t} \varphi_{2} \text { or there is some } \\
& 0 \leq t^{\prime}<t \text {, such that } \bar{\rho}^{t^{\prime}} \stackrel{s}{=}_{\nu-t^{\prime}} \varphi_{1} ; \\
& \bar{\rho} \stackrel{s}{=}{ }_{\nu} x \sim 0 \quad \text { iff } \quad \nu(x) \sim 0 \text {; } \\
& \bar{\rho} \mid \stackrel{s}{=}{ }_{\nu} \bigcirc \psi \quad \text { iff }\left.\quad \bar{\rho}\right|^{o}{ }_{\nu} \psi
\end{aligned}
$$

$\stackrel{o}{=}$ in the last clause is defined for all formulas, except TS. $\psi$ and $\bigcirc \psi$, as:

$$
\left.\bar{\rho}\right|^{=}{ }_{\nu} \psi \quad \text { iff } \text { for every } t \in \bar{I}(1), \bar{\rho}^{t} \stackrel{s}{=}_{\nu-t} \psi
$$

Moreover,

$$
\begin{aligned}
& \bar{\rho} \stackrel{o}{=}_{\nu} T S . \psi \quad \text { iff } \bar{\rho} \stackrel{o}{=}_{T S(\nu)} \psi \text {; } \\
& \bar{\rho} \supseteqq{ }_{\nu} \bigcirc \psi \quad \text { iff } \bar{\rho}^{l(\bar{I}(2))} \stackrel{\mathcal{S}}{=}_{\nu-l(\bar{I}(2))} \psi \text {; }
\end{aligned}
$$

Notice, in particular, the Until formula with bound $\leq x+\epsilon$, which arises from the need to check a bounded Until formula during an entire (left-open) interval as discussed in the example in Section 3. In the remainder we use the notation $\stackrel{i t}{=}, i t \in\{s, o\}$ to denote the following. $\psi_{1} \stackrel{i t}{=} \psi_{2}$ iff for every $\psi_{1}$-fine and $\psi_{2}$-fine timed state sequence $\bar{\rho}$, and every $\nu, \bar{\rho} \stackrel{i t}{=}{ }_{\nu} \psi_{1} \Longleftrightarrow \bar{\rho} \stackrel{i t}{=}{ }_{\nu} \psi_{2}$. If conditions on the timer valuation $\nu$ are mentioned besides the expression $\psi_{1} \stackrel{i t}{\equiv} \psi_{2}$, it means that satisfaction is only identical for timer valuations $\nu$ that satisfy those criteria. We also write $\psi_{1} \stackrel{s, o}{\equiv} \psi_{2}$ to express that both $\psi_{1} \stackrel{s}{\equiv} \psi_{2}$ and $\psi_{1} \stackrel{o}{\equiv} \psi_{2}$. 
The goal is to rewrite $\mathrm{MITL}_{\leq}$formulas into disjunctive temporal normal form, i.e., into a formula of the form

$$
\bigvee_{i} T S_{i} \cdot\left(\pi_{i} \wedge \xi_{i} \wedge \bigcirc \psi_{i}\right)
$$

a disjunction of terms consisting of a timer set operation $T S_{i}$ applied to the conjunction of a propositional formula $\pi_{i}$, timer conditions $\xi_{i}$ and another MITL $\leq$ formula $\psi_{i}$ behind a Next operator. When the tableau automaton is constructed, this corresponds to a collection of transitions labelled with the timer set operation $T S_{i}$ to locations labelled with propositional symbols matching $\pi_{i}$, timer conditions matching $\xi_{i}$ and whose outgoing transitions will be derived from $\psi_{i}$. For instance the formula $p \mathrm{U}_{\leq 5} q$ has an $(\mid \stackrel{s}{=})$ equivalent formula $([x:=5] . q) \vee([x:=$ $\left.5] .\left(p \wedge x>0 \wedge \bigcirc p \mathrm{U}_{\leq x} q\right)\right)$ in disjunctive temporal normal form.

We mention some of the equivalences on which the normal form rewriting procedure is based. The numbers besides the equivalences mention the rules of Table [3, introduced in the next subsection, to which they correspond.

Instantiating Timers These equivalences suggest how to use timers to check timing constraints expressed by formulas.

$$
\begin{gathered}
\varphi_{1} \mathrm{U}_{\leq d} \varphi_{2} \stackrel{\stackrel{s}{\equiv}}{=}[x:=d] .\left(\varphi_{1} \mathrm{U}_{\leq x} \varphi_{2}\right) \\
\varphi_{1} \mathrm{U}_{\leq d} \varphi_{2} \stackrel{o}{=}[x:=d] .\left(\varphi_{1} \mathrm{U}_{\leq x+\epsilon} \varphi_{2}\right)
\end{gathered}
$$

Unfolding These equivalences are used to separate constraints on the current interval from constraints on the remainder of the state sequence.

$$
\begin{aligned}
& \varphi_{1} \mathrm{U}_{\leq x} \varphi_{2} \stackrel{s, o}{\equiv} \varphi_{2} \vee\left(x>0 \wedge \varphi_{1} \wedge \bigcirc \varphi_{1} \mathrm{U}_{\leq x} \varphi_{2}\right) \\
& \varphi_{1} \mathrm{U}_{\leq x+\epsilon} \varphi_{2} \stackrel{s, o}{\equiv} \varphi_{2} \vee\left(x \geq 0 \wedge \varphi_{1} \wedge \bigcirc \varphi_{1} \mathrm{U}_{\leq x+\epsilon} \varphi_{2}\right) \\
& \varphi_{1} \mathrm{~V}_{\leq d} \varphi_{2} \stackrel{s}{\equiv}\left(\varphi_{2} \wedge \varphi_{1}\right) \vee[y:=d] .\left(\varphi_{2} \wedge \bigcirc \varphi_{1} \mathrm{~V}_{\leq y} \varphi_{2}\right) \\
& \varphi_{1} \mathrm{~V}_{\leq d} \varphi_{2} \stackrel{o}{\equiv}\left(\varphi_{2} \wedge \varphi_{1}\right) \vee\left(\varphi_{2} \wedge \bigcirc \varphi_{1} \mathrm{~V}_{<d} \varphi_{2}\right) \\
& \varphi_{1} \mathrm{~V}_{<d} \varphi_{2} \stackrel{s}{\equiv}\left(\varphi_{2} \wedge \varphi_{1}\right) \vee[y:=d] .\left(\varphi_{2} \wedge \bigcirc \varphi_{1} \mathrm{~V}_{<y} \varphi_{2}\right) \\
& \varphi_{1} \mathrm{~V}_{<y} \varphi_{2} \stackrel{s, o}{\equiv}\left(\varphi_{1} \wedge \varphi_{2}\right) \vee y \leq 0 \vee\left(\varphi_{2} \wedge \bigcirc \varphi_{1} \mathrm{~V}_{<y} \varphi_{2}\right) \\
& \varphi_{1} \mathrm{~V}_{\leq y} \varphi_{2} \stackrel{s, o}{=}\left(\varphi_{1} \wedge \varphi_{2}\right) \vee y<0 \vee\left(\varphi_{2} \wedge \bigcirc \varphi_{1} \mathrm{~V}_{\leq y} \varphi_{2}\right)
\end{aligned}
$$

Absorbtion These equivalences enable one to use only a finite number of timers.

$$
\begin{gathered}
\varphi_{1} \mathrm{U}_{\leq x} \varphi_{2} \wedge \varphi_{1} \mathrm{U}_{\leq d} \varphi_{2} \stackrel{s, o}{\equiv} \varphi_{1} \mathrm{U}_{\leq x} \varphi_{2} \text { if } \nu(x) \leq d \\
\varphi_{1} \mathrm{~V}_{<d} \varphi_{2} \wedge \varphi_{1} \mathrm{~V}_{\leq y} \varphi_{2} \stackrel{s}{\equiv} \varphi_{1} \mathrm{~V}_{<d} \varphi_{2} \text { if } \nu(y)<d \\
\varphi_{1} \mathrm{~V}_{\leq y} \varphi_{2} \wedge[y:=d] .\left(\varphi_{1} \mathrm{~V}_{<y} \varphi_{2} \stackrel{s}{\equiv}[y:=d] . \varphi_{1} \mathrm{~V}_{<y} \varphi_{2} \text { if } \nu(y)<d\right.
\end{gathered}
$$

Before we present the normal form procedure, we introduce some more notation to help in the transition from formulas to automata. We identify sets of formulas with the conjunction of the formulas in the set, the empty set being the formula true. We use a triple $\langle T S$, Now, Next $\rangle$ to denote the formula $T S$. (Now $\wedge \bigcirc$ Next). A set of such triples is associated with the disjunction of the corresponding formulas. 


\begin{tabular}{|c|c|c|c|}
\hline & CASE $\psi=$ & CONDITION & $\Phi \cup\{\langle T S$, Now $\cup\{\psi\}$, Next $\rangle\}$ REDUCES TO: \\
\hline C.1 & true & & $\Phi \cup\{\langle T S$, Now, Next $\rangle\}$ \\
\hline C.2 & false & & $\Phi$ \\
\hline C.3 & $\psi_{1} \vee \psi_{2}$ & & $\begin{array}{l}\Phi \cup\left\{\left\langle T S, \text { Now } \cup\left\{\psi_{1}\right\}, \text { Next }\right\rangle\right. \\
\left.\left\langle T S, \text { Now } \cup\left\{\psi_{2}\right\}, \text { Next }\right\rangle\right\}\end{array}$ \\
\hline C. 4 & $\psi_{1} \wedge \psi_{2}$ & & $\Phi \cup\left\{\left\langle T S\right.\right.$, Now $\cup\left\{\psi_{1}, \psi_{2}\right\}$, Next $\left.\rangle\right\}$ \\
\hline C.5 & $\varphi_{1} \mathrm{U}_{\leq d} \varphi_{2}$ & $\begin{array}{l}\Xi^{\psi} \cap \text { Now } \neq \varnothing \text { or } \\
\varphi_{2} \in \text { Now }\end{array}$ & $\Phi \cup\{\langle T S$, Now, Next $\rangle\}$ \\
\hline C. 6 & $\varphi_{1} \mathrm{U}_{\leq x_{\psi}} \varphi_{2}$ & & $\begin{array}{l}\Phi \cup\left\{\left\langle T S, \text { Now } \cup\left\{\varphi_{2}\right\}, \text { Next }\right\rangle\right. \\
\left.\left\langle T S, \text { Now } \cup\left\{x>0, \varphi_{1}\right\}, \text { Next } \cup\{\psi\}\right\rangle\right\}\end{array}$ \\
\hline C.7 & $\varphi_{1} \mathrm{U}_{\leq x}+\epsilon \varphi_{2}$ & & $\begin{array}{l}\Phi \cup\left\{\left\langle T S, \text { Now } \cup\left\{\varphi_{2}\right\}, \text { Next }\right\rangle\right. \\
\left.\left\langle T S, \text { Now } \cup\left\{x \geq 0, \varphi_{1}\right\}, \text { Next } \cup\{\psi\}\right\rangle\right\}\end{array}$ \\
\hline C.8 & $\varphi_{1} \mathrm{~V}_{<y_{\psi}} \varphi_{2}$ & & $\begin{array}{l}\Phi \cup\left\{\left\langle T S, \text { Now } \cup\left\{\varphi_{1}, \varphi_{2}\right\}, \text { Next }\right\rangle,\langle T S, \text { Now } \cup\right. \\
\left.\left.\left\{y_{\psi} \leq 0\right\}, \text { Next }\right\rangle,\left\langle T S, \text { Now } \cup\left\{\varphi_{2}\right\}, \text { Next } \cup\{\psi\}\right\rangle\right\}\end{array}$ \\
\hline C.9 & $\varphi_{1} \mathrm{~V}_{\leq y_{\psi}} \varphi_{2}$ & & $\begin{array}{l}\Phi \cup\left\{\left\langle T S, \text { Now } \cup\left\{\varphi_{1}, \varphi_{2}\right\}, \text { Next }\right\rangle,\langle T S, \text { Now } \cup\right. \\
\left.\left.\left\{y_{\psi}<0\right\}, \text { Next }\right\rangle,\left\langle T S, \text { Now } \cup\left\{\varphi_{2}\right\}, \text { Next } \cup\{\psi\}\right\rangle\right\} \\
\end{array}$ \\
\hline S.1 & $\varphi_{1} \mathrm{U}_{\leq d} \varphi_{2}$ & $\begin{array}{l}\Xi^{\psi} \cap \text { Now }=\varnothing \\
\text { and } \varphi_{2} \notin \text { Now }\end{array}$ & $\Phi \cup\left\{\left\langle T S[x:=d], N o w \cup\left\{\varphi_{1} \cup_{\leq x_{\psi}} \varphi_{2}\right\}, N e x t\right\rangle\right\}$ \\
\hline $\mathrm{S} .2$ & $\varphi_{1} \mathrm{~V}_{\leq d} \varphi_{2}$ & & $\begin{array}{l}\Phi \cup\left\{\left\langle T S, \text { Now } \cup\left\{\varphi_{2}, \varphi_{1}\right\}, \text { Next }\right\rangle,\langle T S[y:=d]\right. \\
\left.\left.N o w \backslash \Xi^{\psi} \cup\left\{\varphi_{2}\right\}, \text { Next } \cup\left\{\varphi_{1} \vee_{\leq y_{\psi}} \varphi_{2}\right\}\right\rangle\right\}\end{array}$ \\
\hline S.3 & $\varphi_{1} \mathrm{~V}_{<d} \varphi_{2}$ & $\begin{array}{l}\varphi_{1} \mathrm{~V}_{\leq y_{\psi}} \varphi_{2} \notin \text { Now or } \\
y_{\psi}:=d \in T S \\
\varphi_{1} \mathrm{~V}_{\leq y_{\psi}} \varphi_{2} \in \text { Now } \\
y_{\psi}:=d \notin T S \\
\end{array}$ & $\begin{array}{l}\Phi \cup\left\{\left\langle T S, N o w \cup\left\{\varphi_{2}, \varphi_{1}\right\}, \text { Next }\right\rangle,\langle T S[y:=d],\right. \\
\left.\left.N o w \backslash \Xi^{\psi} \cup\left\{\varphi_{2}\right\}, N e x t \cup\left\{\varphi_{1} \vee_{<y_{\psi}} \varphi_{2}\right\}\right\rangle\right\} \\
\Phi \cup\left\{\left\langle T S, N o w \cup\left\{\varphi_{2}, \varphi_{1}\right\}, N e x t\right\rangle,\langle T S\lfloor y:=d],\right. \\
\left.\left.N o w \backslash \Xi^{\psi} \cup\left\{\varphi_{1} \vee_{<y_{\psi}} \varphi_{2}\right\}, \text { Next }\right\rangle\right\}\end{array}$ \\
\hline O.1 & $\varphi_{1} \mathrm{U}_{\leq d} \varphi_{2}$ & $\begin{array}{l}\Xi^{\psi} \cap \text { Now }=\varnothing \\
\text { and } \varphi_{2} \notin \text { Now }\end{array}$ & $\Phi \cup\left\{\left\langle T S[x:=d]\right.\right.$, Now $\left.\left.\cup\left\{\varphi_{1} \mathrm{U}_{\leq x}{ }_{\psi}+\epsilon \varphi_{2}\right\}, N e x t\right\rangle\right\}$ \\
\hline 0.2 & $\varphi_{1} \mathrm{~V}_{\leq d} \varphi_{2}$ & & $\begin{array}{l}\Phi \cup\left\{\left\langle T S, \text { Now } \cup\left\{\varphi_{2}, \varphi_{1}\right\}, \text { Next }\right\rangle\right. \\
\left.\left\langle T S, \text { Now } \cup\left\{\varphi_{2}\right\}, \text { Next } \cup\left\{\varphi_{1} \vee_{<d} \varphi_{2}\right\}\right\rangle\right\}\end{array}$ \\
\hline 0.3 & $\varphi_{1} \mathrm{~V}_{<d} \varphi_{2}$ & & $\begin{array}{l}\Phi \cup\left\{\left\langle T S, \text { Now } \cup\left\{\varphi_{2}, \varphi_{1}\right\}, \text { Next }\right\rangle\right. \\
\left.\left\langle T S, \text { Now } \cup\left\{\varphi_{2}\right\}, \text { Next } \cup\left\{\varphi_{1} \vee_{<d} \varphi_{2}\right\}\right\rangle\right\}\end{array}$ \\
\hline
\end{tabular}

Fig. 3. Rewrite rules for singular (S.n), open (O.n) and common rules (C.n)

\subsection{The Normal Form Procedure}

We now introduce a procedure that rewrites extended MITL $\leq$ formulas into normal form, and is used as an integral part of the tableau construction.

Definition 5. Let $\Psi$ be a set of formulas, then the normal form $N F(i t, \Psi)$ of $\Psi$ for an interval of type it is computed with the following procedure. Let $P_{0}=$ $\{\langle\varnothing, \Psi, \varnothing\rangle\}$. Then as long as one of the rewrite rules (C.1-9, S.1-4 for it $=s$ and C.1-9, O.1-3 for it =o) of Table 3 applies to any of the terms in $P_{n}$, apply one to a term to obtain $P_{n+1}$. The normal form $N F(i t, \Psi)$ is obtained from $P_{n}$ when no more rule applies.

It is easy to check that the procedure ends and the resulting formula is in normal form. If no more rule applies, then for every term, Now contains only propositions and timer conditions. Moreover, the resulting formula is equivalent w.r.t the interpretation $\stackrel{i t}{=}$ :

Lemma 1. Let $\Psi \subseteq \operatorname{cl}(\varphi)$ for some $\varphi \in \mathrm{MITL}_{\leq}, \nu$ be a timer valuation such that $\nu\left(x_{\psi}\right) \leq d$ for every Until or Release formula $\psi \in \operatorname{cl}(\varphi)$ with bound $d$, and $\bar{\rho}$ a timed state sequence with interval sequence $\bar{I}$ that is fine for all basic subformulas 


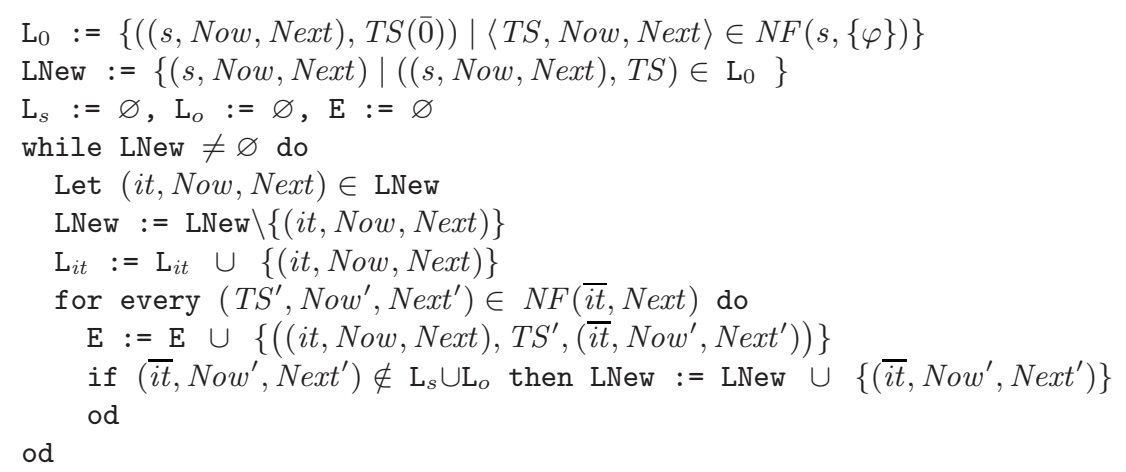

Fig. 4. Algorithm for constructing the locations and edges of the tableau automaton.

of $\Psi$. If $\bar{\rho} \stackrel{i t}{=}{ }_{\nu} \Psi$, then there is some term $\langle i t, T S, N o w, N e x t\rangle \in N F(i t, \Psi)$ such that $\bar{\rho} \stackrel{i t}{=}_{T S(\nu)}$ Now, and $\bar{\rho} \stackrel{i t}{=} T S(\nu) \bigcirc N e x t$.

Proofs have been left out due to space limitations. Details can be found in [7].

\section{Tableau Construction}

The tableau automaton of an $\mathrm{MITL}_{\leq}$formula $\varphi$ is computed as follows. (With $i t \in\{s, o\}$, we use $\overline{i t}$ to denote $o$ if $\overline{i t}=s$ and $s$ if $i t=o$.)

Definition 6. Let $\varphi$ be a basic MITL $\leq$ formula and Prop be the set of atomic propositions that occur in $\varphi$. Then the tableau automaton $A_{\varphi}$ of $\varphi$ is the automaton $\left\langle L_{s}, L_{o}, T, L_{0}, Q, T C, E\right\rangle$ over the alphabet $2^{\text {Prop }}$, where

- $T$ is the set of all timers $x_{\psi}$ for every Until or Release formula $\psi$ that occurs as a syntactic subformula of $\varphi$.

- The locations $\left(L_{s}, L_{o}\right)$, initial extended locations $\left(L_{0}\right)$ and transitions $(E)$ are computed by the procedure depicted in Fig. 4. The locations $\ell \in L_{s} \cup L_{o}$ are triples (it, Now, Next). The first item of the location $\ell$ is denoted by it $(\ell)$, the second by $N o w(\ell)$ and the last by $N \operatorname{ext}(\ell)$.

- $Q(\ell)=\left\{\sigma \in 2^{\text {Prop }} \mid \forall_{p \in \text { Prop }} p \in\right.$ Now $(\ell) \Rightarrow p \in \sigma, \neg p \in$ Now $\left.(\ell) \Rightarrow p \notin \sigma\right\}$. That is, a location $\ell$ is labelled with all sets of propositions that are consistent with the atomic propositions and the negated atomic propositions in Now $(\ell)$.

- $T C(\ell)=T C o n d(T) \cap N o w(\ell)$, the location is labelled with all timer conditions in Now $(\ell)$.

Example With a prototype implementation of the algorithm, the following example was produced for the formula $\square_{\leq 100} \vee_{\leq 5} p=$ falseV $_{\leq 100}\left(\operatorname{trueU}_{\leq 5} p\right)$. We arrive at the automaton represented in Fig. 5. Only the formulas in the Now set of the locations have been depicted. The (two) initial extended locations are 


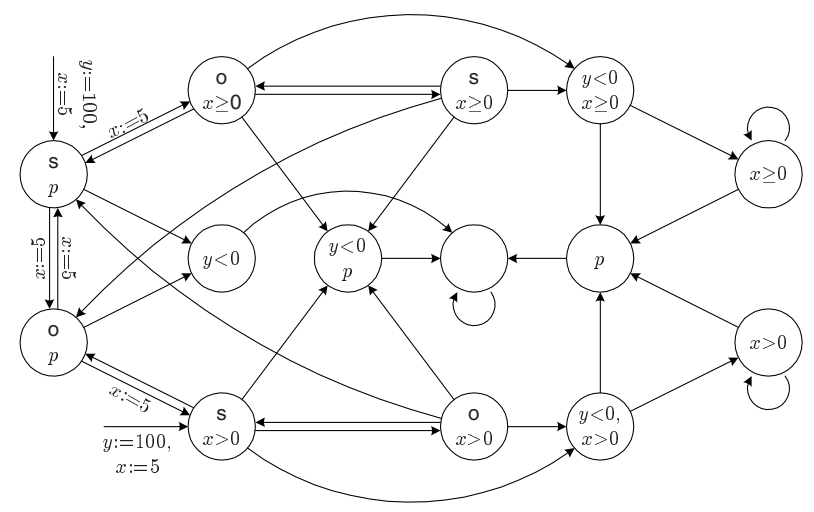

Fig. 5. Example tableau automaton of the formula $\square_{\leq 100} \oslash_{\leq 5} p$

represented by an arrow not originating from any location leading to the initial location and labelled with a timer setting that yields the initial timer valuation when applied to the timer valuation that assigns 0 to every timer. There is a timer associated with the formula $\vartheta_{\leq 5} p$ named $x$ and a timer associated with the formula $\square_{\leq 100} \vartheta_{\leq 5} p$ named $y$. Singular locations are labelled with 's', open location are labelled with ' $\mathrm{o}$ '. To simplify the figure, locations without ' $\mathrm{s}$ ' or ' $\mathrm{o}$ ' represent two locations, a singular one and an open one, when they are labelled identically (a straightforward optimisation opportunity for the algorithm); without the automaton has 22 locations and 46 edges.

That the presented algorithm is correct, is stated by the following theorem.

Theorem 1. Let $\varphi$ be a basic $\mathrm{MITL}_{\leq}$formula and $A_{\varphi}$ the corresponding tableau automaton, then for every timed state sequence $\bar{\rho}, A_{\varphi}$ accepts $\bar{\rho}$ iff $\bar{\rho} \models \varphi$.

Details and proof can be found in [7].

\section{Conclusions}

We have presented an on-the-fly tableau construction for the fragment $\mathrm{MITL}_{\leq}$ of Mitl. It employs the introduction of explicit timers and timer set operators into the logic, as well as a Next operator as in [8], but represents a non/trivial improved of [8] by the ability to deal with arbitrary interval sequences, and thereby lifts the restrictions to special intervals and makes it applicable to standard timed systems. In [4 it has been shown that the construction of tableaux for $\mathrm{MITL}_{0, \infty}$, a slightly different fragment of MitL, is PSPACE-complete, and this result can be adapted to the case of $\mathrm{MITL}_{\leq}$. Thus, the theoretical worstcase complexity of our construction is the same as that of the construction in [4]. Being on-the-fly, this algorithm should give much smaller tableaux in practice. Moreover, we have found a small deficiency of the construction of [24]. It is believed that it can be resolved with concepts from our algorithm. Extension of the logic with liveness properties, strict bounds and lower bounds instead of upper 
bounds are straightforward, but have been left out for clarity. We further think that this work can form the basis for further extensions towards weakly monotonic models of time, often found in interleaving semantics for timed systems and tools such as UPPAAL 11. Also, optimisations that have been developed for (on-the-fly) tableau constructions in the untimed case (see e.g. [56[9]) might be applicable to our algorithm.

\section{References}

1. L. Aceto, A. Burgueño, and K. Larsen. Model checking via reachability testing for timed automata. In B. Steffen, editor, Proc. of Tools and Algorithms for Construction and Analysis of Systems, 4th Int. Conf., TACAS '98, Lisbon, Portugal, March 28 - April 4 1998, LNCS Vol. 1384, pages 263-280, 1998. Springer Verlag.

2. R. Alur. Techniques for Automatic Verification of Real-Time Systems. PhD thesis, Stanford University, 1991.

3. R. Alur and D.L. Dill. A theory of timed automata. Theoretical Computer Science, 126:183-235, 1994.

4. R. Alur, T. Feder, and T. Henzinger. The benefits of relaxing punctuality. Journal of the ACM, 43(1):116-146, January 1996.

5. M. Daniele, F. Giunchiglia, and M. Vardi. Improved automata generation for linear temporal logic. In N. Halbwachs and D. Peled, editors, Proc. of Computer Aided Verification: 11th Int. Conf., CAV'99, Trento, Italy, July 6-10, 1999, LNCS 1633, pages 249-260. Springer Verlag, 1999.

6. K. Etessami and G. Holzman. Optimising Büchi automata. In C. Palamidessi, editor, Proc. of the 11th Int. Conf. on Concurrency Theory (CONCUR'2000), Pennsylvania State University, Pennsylvania, USA, August 22-25, 2000, LNCS 1877, pages 153-167, 2000. Springer Verlag.

7. M.C.W. Geilen. Formal Techniques for Verification of Complex Real-Time Systems. PhD thesis, Eindhoven University of Technology, 2002.

8. M.C.W. Geilen and D.R. Dams. An on-the-fly tableau construction for a real-time temporal logic. In M. Joseph, editor, Proc. of the 6th Int. Symp. on Formal Techniques in Real-Time and Fault-Tolerant Systems, FTRTFT2000, 20-22 September 2000 Pune, India, LNCS 1926, pages 276-290, 2000. Springer Verlag.

9. R. Gerth, D. Peled, M. Vardi, and P. Wolper. Simple on-the-fly automatic verification of linear temporal logic. In Proc. IFIP/WG6.1 Symp. Protocol Specification Testing and Verification (PSTV95), Warsaw Poland, pages 3-18. Chapman \& Hall, June 1995.

10. T. Henzinger. It's about time: Real-time logics reviewed. In D. Sangiorgi and R. de Simone, editors, Proc. of the 9th Int. Conf. on Concurrency Theory (CONCUR'98), Nice, France, 1998, LNCS 1466, pages 439-454, 1998. Springer Verlag.

11. K. Larsen, P. Pettersson, and W. Yi. UPPAAL in a nutshell. Int. Journal on Software Tools for Technology Transfer, 1(1-2):134-152, October 1997.

12. O. Lichtenstein and A. Pnueli. Checking that finite state concurrent programs satisfy their linear specification. In Proc. of 12th Annual ACM Symp. Principles of Programming Languages, pages 97-107. ACM SIGACT/SIGPLAN, 1985.

13. A. Pnueli. The temporal logic of programs. In Proc. of the 18th Ann. Symp. on Found. of Computer Science, pages 46-57. IEEE Computer Society Press, 1977.

14. M.Y. Vardi and P. Wolper. An Automata-Theoretic approach to automatic program verification. In Proc. of Logic in Computing Science, 1986. 